\title{
First Human Evaluation of Endothelial Healing after a Pipeline Flex Embolization Device with Shield Technology Implanted in Posterior Circulation Using Optical Coherence Tomography
}

\author{
Boris Pabón Guerrero, $\mathrm{MD}^{1}$, Carlos Díaz Pacheco, $\mathrm{MD}^{1,2}$, Ahmed Saied, $\mathrm{MD}^{3,4}$, Krishna Joshi, $\mathrm{MD}^{3}$, \\ Claudio Rodríguez, $\mathrm{MD}^{5}$, Mario Martínez-Galdámez, $\mathrm{MD}^{5}$, Demetrius K. Lopes, $\mathrm{MD}^{3}$ \\ ${ }^{1}$ Angioteam, Angiosur, Medellín, Colombia \\ ${ }^{2}$ Department of Radiology, Universidad de Antioquia, Medellín, Colombia \\ ${ }^{3}$ Department of Neurological Surgery, Rush University Medical Center, Chicago, IL, USA \\ ${ }^{4}$ Department of Neurology, Mansoura University, Mansoura, Egypt \\ ${ }^{5}$ Interventional Neuroradiology Unit, Hospital Universitario Fundación Jiménez Díaz, Madrid, Spain
}

A 64-year-old female presented with an incidentally-discovered right posterior inferior cerebral artery (PICA) aneurysm, initially treated in 2015 by simple coiling. Follow-up demonstrated significant coil compaction that required retreatment. Retreatment was done uneventfully using a Pipeline embolization device (PED) shield deployed starting from the basilar artery and ending at the V4 segment of the vertebral artery. Eight-weeks post-deployment, a follow-up digital subtraction imaging (DSA) and intravascular imaging with optical coherence tomography were obtained. The intravascular imaging demonstrated that the flow diverter had good wall apposition and concentric neointimal growth over the braid with exception to the areas that the PED was not in contact with the endothelial wall, such as at the right PICA ostium and at the vertebrobasilar junction. The entire procedure was safe, and the patient had no complications, In this article, we describe for the first time the assessment of the status of endothelial "healing" of the PED shield at 8-weeks.

Key Words: Tomography, Optical coherence; Flow diverter; Pipeline shield

\section{INTRODUCTION}

Flow diversion devices are widely used for the treatment of intracranial aneurysms. The safety and efficacy of the first generation Pipeline embolization device (PED; Medtronic Neurovascular, Irvine, $\mathrm{CL}$, USA) have been demonstrated in many clinical studies where most of the adverse events were ischemic. PED Flex with Shield Technology (PED Shield) was introduced using shield technology to decrease its thrombogenicity. ${ }^{2}$

Optical coherence tomography (OCT) is an intravascular imaging technique ideal to evaluate the healing of vascular implants since it provides a histopathological view of the device and vessel wall. ${ }^{3}$ It is unclear the impact of surface modification on the neointimal formation over the implanted device. In this article, we describe the status of the

\section{Correspondence to:}

Demetrius K. Lopes, MD

Department of Neurological Surgery, Rush University Medical Center, 1725 W. Harrison St, Professional Building Suite 855, Chicago, IL 60612, USA Tel: +1-312-942-1854

Fax: +1-312-942-2176

E-mail: Demetrius_Lopes@Rush.edu

Received: June 16, 2018

Revised: July 16, 2018

Accepted: July 17, 2018
Copyright $\odot 2018$ Korean Society of Interventional Neuroradiology This is an Open Access article distributed under the terms of the Creative Commons Attribution Non-Commercial License (http://creativecommons.org/licenses/by-nc/3.0) which permits unrestricted non-commercial use, distribution, and reproduction in any medium, provided the original work is properly cited.

pISSN 2093-9043 eISSN 2233-6273 
neointima development over the new PED shield at 8-weeks post-implantation.

\section{CASE REPORT}

A 64-year-old female presented with an incidentally-discovered right posterior inferior cerebellar artery (PICA) aneurysm initially treated by coiling. Follow-up demonstrated recanalization with significant coil compaction that required retreatment. We thought that further coiling might compromise the flow through the PICA and that it would be very probable in the future to have a similar recanalization with the same treatment strategy with coils. The PED Shield was uneventfully used from the basilar to vertebral artery across the origin of PICA and the coiled aneurysm. The patient received dual antiplatelet therapy one week before the procedure. At 8-weeks, clinical and imaging follow-ups were obtained. There were no thromboembolic complications; on digital subtraction imaging (DSA), the aneurysm was stable with preserved blood flow through the PICA (Fig. 1). OCT imaging was planned to evaluate the degree of neointimal growth over the stent to assess the possibility of removal of one of the antiplatelet agents in favor of PICA aneurysm occlusion.

\section{OCT image technique}

After obtaining the patient's consent, our endovascular access used a radial artery approach. A 6 F Sofia distal access catheter (Microvention/Terumo, Tustin, CA, USA) over a 0.014" Traxcess microguidewire (Microvention/Terumo) was advanced through the right vertebral artery into the basilar artery using the standard technique. During the procedure, we used a continuous saline flush in our catheters. The patient was heparinized (5,000 IU) and under general anesthesia.

The C7 Dragonfly, a monorail 2.7 F OCT catheter (St. Jude Medical Inc., St. Paul, MN, USA), was advanced inside the distal access catheter (Fig. 1). The OCT image was generated as the optical fiber in the catheter moved using an automated pullback system during simultaneous contrast infusion at a rate of approximately $3 \mathrm{~mL} / \mathrm{sec}$. Imaging of about a $5 \mathrm{~cm}$ segment of the vessel was achieved with a $15 \mathrm{~mL}$ contrast injection necessary to obtain clear images. The OCT system C7 Dragonfly Intravascular Imaging Catheter is more rigid compared to conventional neurointervention systems and potentially traumatic if not carefully navigated. The fiber optic inside the catheter is sensitive to manipulation and presents a risk of fracture during navigation in tortuous vessels, but does not present a risk of catheter fracture or intravascular migration. There were no complications during the procedure.

\section{OCT image interpretation}

OCT images demonstrated excellent visualization of PED Shield struts, device wall apposition, and neointima development. Concentric neointimal growth over the PED Shield braid was evident in all areas that the device was well apposed against the vessel wall. No neointima was seen at this 8-week follow-up in areas of device malapposition as well as areas with no contact with the endothelial wall such as the vertebra-basilar junction, perforator, and PICA origin. No thrombi were found on the exposed part of the PED Shield. Normal arterial wall imaging was evident both proximal and distal to the flow diverter. No dissection, intimal tear, or stent stenosis were found (Fig. 1).

\section{DISCUSSION}

The PED Shield, the third generation of PED, has been introduced to potentially improve its thrombogenic profile. In comparison to other flow diverters, the PED Shield showed a lower thrombogenic profile in vitro, ${ }^{4}$ ex vivo model, ${ }^{5}$ and clinical studies. ${ }^{2}$

OCT imaging has been used with flow diverters in cadaveric $^{6}$ and animal studies where the PED Shield was less likely to form acute thrombus on its surface, ${ }^{7}$ and has a tendency for earlier and evenly-distributed concentric neointimal formation compared to other stents and flow diverters. ${ }^{8}$

Neurovascular OCT imaging faces multiple technical challenges and is currently off label in the USA. The current commercially-available OCT technology requires perfect timing of contrast injection and catheter pull back. The collateral circulation through the circle of Willis quickly will "contaminate" the OCT image with blood. This limits the location and length of OCT imaging that can be obtained. The second challenge is the tortuosity of the cerebral blood vessels. ${ }^{6}$ The third potential challenge is the profile of the current system, which makes the possibility injury to the blood vessels and complications that may potentially eliminate the benefit of OCT diagnostic imaging. ${ }^{9}$

Previous reports raise the concern that clot formation can occur at the level of the exposed device braid at the ostia of 

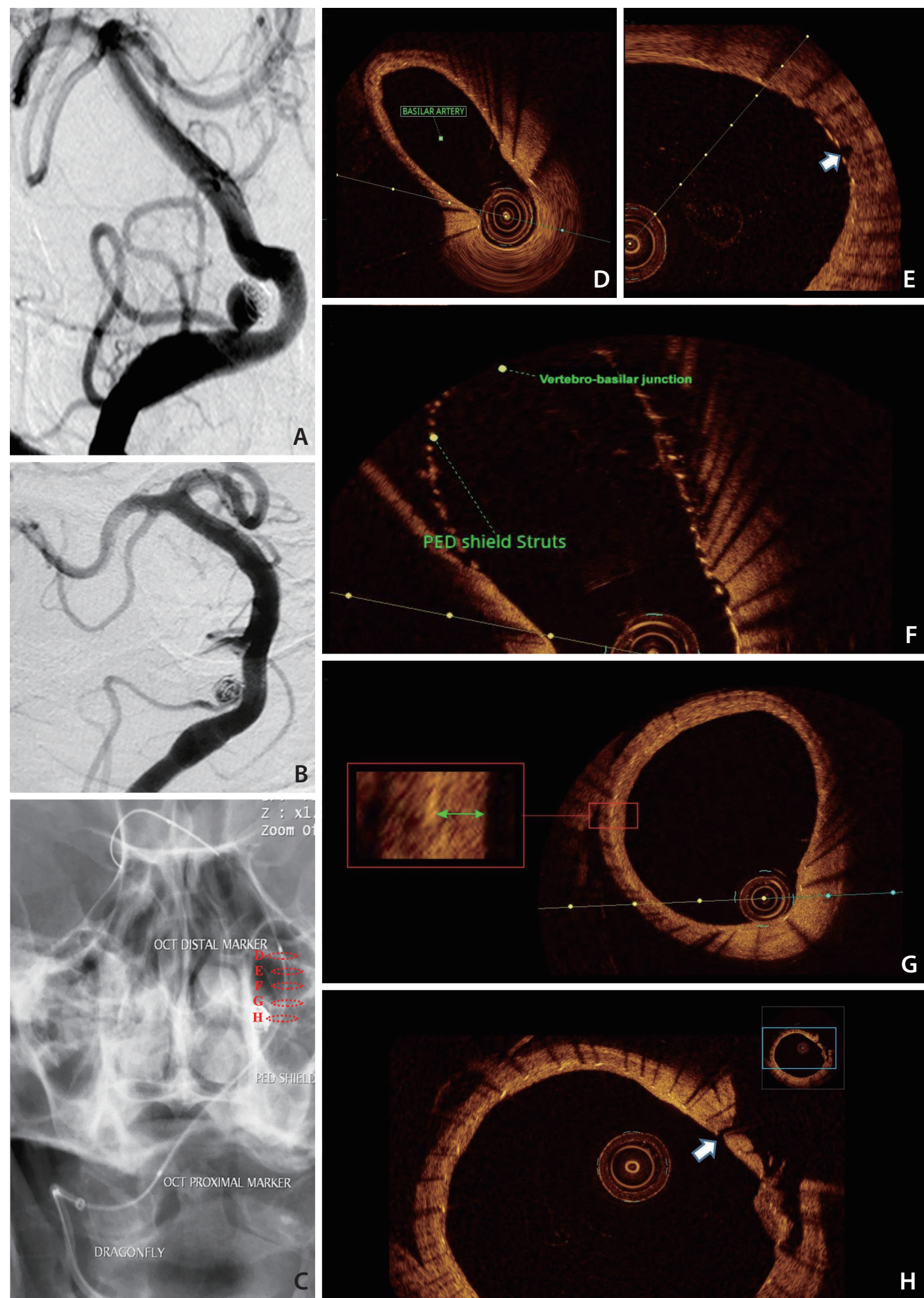

Fig. 1. Conventional angiography of the vertebra-basilar system antero-posterior view. (A) Recanalized right PICA aneurysm with significant coil compaction. (B) PICA aneurysm at 8-weeks follow-up of the PED shield deployment. (C) Dragonfly OCT catheter safely advanced along distal access catheter to the basilar artery after 8-weeks of PED Shield deployment. Dashed circles in (C) correspond to the cross sections from the OCT acquisition from within the vertebra-basilar system. (D-H) PED Shield struts, device wall apposition, and neointima development in the basilar artery (D). No neointima was seen over the basilar perforator (white arrow) (E) or the vertebra-basilar junction (F). (G) PED Shield struts, device wall apposition, and neointima development (green arrow) in the vertebral artery. (H) No neointima was seen over the PICA origin (white arrow). PICA, posterior inferior cerebral artery; PED, Pipeline embolization device; OCT, optic coherence tomography. 
side branches. ${ }^{7}$ This may be a very important differential for devices with surface modification. Another very convincing finding was the relatively early (8-weeks post-implantation) full endothelialization seen for the well-apposed PED Shield to the vessel wall. Surface modified devices may have an earlier neointimal formation confirming reports from an animal study suggesting that the PED shield has faster endotheliazation than a regular PED. ${ }^{8}$

OCT intravascular imaging is a promising tool for assessment of intracranial stents and flow diverters. We foresee many improvements on this technology that would allow us in the near future to incorporate intravascular imaging in our pre-, intra-, and post-procedural evaluation in an effort to improve even more our outcomes in neurointervention. OCT pre-procedural assessment can provide accurate vessel measurement and superior intravascular anatomy visualization in comparison to any other method. Intra-procedural OCT assessment can visualize strut wall apposition, intra-strut tissue prolapse, stent measurements post-deployment, microthrombi on the strands, and assessment of perforators. Post-procedural follow-up includes evaluation of wall apposition, tissue response (healing), thrombus formation, stent stenosis, dissection, and assessment of the perforators and side branches at their take off. ${ }^{3}$

There are many limitations to the use of commercially-available OCT imaging for neurovascular applications. The off-label use of OCT imaging of the vertebrobasilar system in this case was technically straightforward and safe. The information regarding the status of the endotheliazation of the device is crucial to assist with determination of the course of antiplatelet therapy. The most impressive findings were complete endothelial coverage over all well-opposed PED shield segments that were in contact with the vessel wall at 8-weeks and no thrombi on the exposed portions of the braid at the level of the patent jailed vessels. This could potentially have an impact on the length of dual antiplatelet therapy needed post flow diversion.

\section{REFERENCES}

1. Brinjikji W, Lanzino G, Cloft HJ, Siddiqui AH, Boccardi E, Cekirge $S$, et al. Risk factors for ischemic complications following pipeline embolization device treatment of intracranial aneurysms: results from the IntrePED study. AJNR Am J Neuroradiol 2016;37:1673-1678

2. Martínez-Galdámez M, Lamin SM, Lagios KG, Liebig T, Ciceri EF, Chapot $\mathrm{R}$, et al. Periprocedural outcomes and early safety with the use of the Pipeline Flex Embolization Device with Shield Technology for unruptured intracranial aneurysms: preliminary results from a prospective clinical study. J Neurointerv Surg 2017:9:772-776

3. Tearney G, Regar E, Akasaka T, Adriaenssens T, Barlis P, Bezerra $H G$, et al. Consensus standards for acquisition, measurement, and reporting of intravascular optical coherence tomography studies: a report from the International Working Group for Intravascular Optical Coherence Tomography Standardization and Validation. J Am Coll Cardiol 2012;59:1058-1072

4. Girdhar G, Li J, Kostousov L, Wainwright J, Chandler WL. In-vitro thrombogenicity assessment of flow diversion and aneurysm bridging devices. J Thromb Thrombolysis 2015;40:437-443

5. Hagen MW, Girdhar G, Wainwright J, Hinds MT. Thrombogenicity of flow diverters in an ex vivo shunt model: effect of phosphorylcholine surface modification. J Neurointerv Surg 2016;9:1006-1011

6. Lopes DK, Johnson AK. Evaluation of cerebral artery perforators and the pipeline embolization device using optical coherence tomography. J Neurointerv Surg 2012;4:291-294

7. Marosfoi M, Clarencon F, Langan ET, King RM, Brooks OW, Tamura T, et al. Acute thrombus formation on phosphorilcholine surface modified flow diverters. J Neurointerv Surg 2018;10:406411

8. Matsuda Y, Chung J, Lopes DK. Analysis of neointima development in flow diverters using optical coherence tomography imaging. J Neurointerv Surg 2018;10:162-167

9. Given CA 2nd, Ramsey CN 3rd, Attizzani GF, Jones MR, Brooks $W H$, Bezerra HG, et al. Optical coherence tomography of the intracranial vasculature and Wingspan stent in a patient. BMJ Case Rep 2014;2014:bcr2014011114 